\title{
Article \\ High-Resolution Simulation of Externally Injected Lasers Revealing a Large Regime of Noise-Induced Chaos
}

\author{
Sean P. O’Duill *(D) and Liam P. Barry
}

\author{
Radio and Optical Communications Laboratory, School of Electronic Engineering, Dublin City University, \\ Glasnevin, D09 DX63 Dublin, Ireland; liam.barry@dcu.ie \\ * Correspondence: sean.oduill@dcu.ie
}

Citation: O'Duill, S.P.; Barry, L.P. High-Resolution Simulation of Externally Injected Lasers Revealing a Large Regime of Noise-Induced Chaos. Photonics 2022, 9, 83. https:// doi.org/10.3390/photonics9020083

Received: 29 November 2021

Accepted: 26 January 2022

Published: 31 January 2022

Publisher's Note: MDPI stays neutral with regard to jurisdictional claims in published maps and institutional affiliations.

Copyright: (C) 2022 by the authors. Licensee MDPI, Basel, Switzerland. This article is an open access article distributed under the terms and conditions of the Creative Commons Attribution (CC BY) license (https:// creativecommons.org/licenses/by/ $4.0 /)$.

\begin{abstract}
We present comprehensive numerically simulated scans of the spectral evolution of the output from a single-mode semiconductor laser diode undergoing external light injection. The spectral scans are helpful to understand the different regimes of operation as well as the system evolution between each state: i.e., locked state, four-wave mixing, pulsations, chaos. We find that, when under strong injection, when the injected power equals about half of the laser power, two distinct regions of chaotic behaviour are observed. One of the chaotic regions arises due to the usual period-doubling route to chaos; the other chaotic region is a blurring of what would be higher-order period pulsations whose periodicity is broken by spontaneous emission and the laser spectrum is chaotic. Eliminating spontaneous emission in our simulations confirms the latter chaotic region becomes a region with higher-order pulsations.
\end{abstract}

Keywords: semiconductor lasers; injection-locking; noise; simulation; pulsation; chaos

\section{Introduction}

External laser injection-locking is a curious field of study due to the range of dynamical states that are produced: laser synchronisation; chaos; four-wave mixing (FWM); selfpulsation and associated period-doubling routes to chaos [1-10]. Recently, it was discovered that lasers acting under injection-locking exhibit a strong increase in the potential direct modulation bandwidth, with subsequent studies to unlock the potential of using such strong modulation capability $[9,10]$, with algorithms developed to achieve the optimal injection-locking point [11]. A recent detailed review, in article [4], details optical injection applications including laser synchronisation for phase-sensitive applications and frequency distribution. Injection-locking is also important for stabilizing gain-switched optical pulse sources and for phase stabilization of optical frequency combs [12,13]. External injection of a semiconductor is highlighted in Figure 1, where two lasers are involved and light from the master laser is injected into the slave laser. The optical isolator (Iso.) ensures that no light from the slave laser is injected back into the master laser. In order to observe the phenomena associated with external injection, the central lasing frequency (or wavelength) of both lasers should be similar, within $\pm 25 \mathrm{GHz}$ of each other.

Laser rate equations have been shown to capture the various phenomena of externally injected lasers [1-3,6]. Despite their simplicity, the equations reveal all of the relevant dynamical phenomena of externally injected lasers. An extended treatise in [6], analysing the dynamical regimes of externally injected lasers, is given in this paper, identifying chaotic, pulsation states including Hopf-bifurcations of the pulsation into general period-N $(\mathrm{P}-\mathrm{N})$ pulsation states [6]. One aspect that we notice when solving laser rate equations when spontaneous emission (SE) is included [3] is the absence of higher pulsation states beyond the P-2 pulsation states; this would be predicted when SE is omitted in the analysis. Previous studies attributed SE to be the cause of an observed absence of a period-doubling route to chaos [14]; a more formal analysis [15] examined the behaviour of noise-induced 
chaos when the external-injected laser system was in a delicate high order pulsation state and that study found that SE could induce chaotic behaviour.

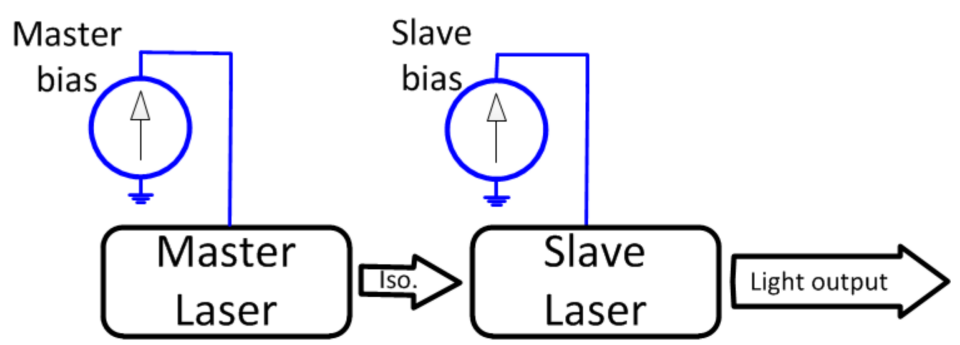

Figure 1. Schematic of external injection laser system. Light from the master laser is coupled to the slave laser, the optical isolator (Iso.) ensures that light from the slave laser does not couple to the master. Depending on the master-slave detuning, master power lever, a whole range of dynamical effects are observed, from injection-locking, four-wave mixing, pulsation and chaos.

In this paper, we present a detailed study of the evolution of the optical spectral output from the injected laser system. We concentrate on varying the detuning frequency between the master and slave lasers and building up the output spectra. The plots are characterised by constant master power, and show FWM, injection-locking, self-pulsations, period-doubling and chaos. We find that only period-2 (P-2) pulsations (i.e., pulsations with double the period of the self-pulsation) are present when we solve the complete rate equations with stochastic SE included. For the first time, to the best of our knowledge, we find a chaotic regime solely created from noise-induced chaos, when SE is removed from the simulation that the region is entirely comprised of high-order pulsations of periodicity P-2 and greater. The conditions to observe this noise-induced chaos regime is when the injection level is quite high, close to $50 \%$ of the slave laser power. The noise-induced chaos regime is entirely separate from the chaotic regime caused by the usual period-doubling route to chaos that remains even when the SE is set to zero. In order to quantify how much SE is needed for noise-induced chaos to exist, we use a value for the SE coupling coefficient into the lasing mode to be $1 \times 10^{-4}$; this value would be at the lower end of the observed values for this parameter [16]. We run our simulations with hypothetical lower values of SE coupling coefficient and that for values $<1 \times 10^{-7}$ that noise-induced chaos still exists.

\section{Materials and Methods}

To conduct this study, we employ the field version of the rate equations for semiconductor lasers. The fundamental derivation of the model is given in [3], though we keep with the complex-valued envelope of the optical field to avoid numerical instability issues when the slave power tends briefly to zero, as can happen with external injection. We implemented the field version of the rate equations before in relation to laser gain-switching [17]; here, we just concentrate on DC biasing of the lasers.

$$
\begin{gathered}
\frac{d N}{d t}=\frac{I_{b i a s}}{e V}-R(N)-\frac{a\left(N-N_{0}\right)}{1+\varepsilon_{N L}|E|^{2}}|E|^{2}+F_{N} \\
\frac{d E}{d t}=\frac{\left(1-j \alpha_{H}\right)}{2}\left[\frac{a\left(N-N_{0}\right)}{1+\varepsilon_{N L}|E|^{2}}-\frac{1}{\tau_{p}}\right] E+k_{c} E_{\text {inj }} \exp \left(j 2 \pi f_{D} t\right)+F_{E}
\end{gathered}
$$

where all of the symbols have their usual meaning and are defined in Table 1 . The carrier density is given by $N$ and $E$ is the envelope of the optical field and is related to the photon density in the laser. The first term on the right-hand side of (1) is given by the electric bias current flowing into the laser, where $I_{\text {bias }}=60 \mathrm{~mA}$ throughout for this study. Carrier recombination is given by $R(N)=A N+B N^{2}+C N^{3}$ for nonradiative, bimolecular and Auger recombination, respectively; the third term represents stimulated emission. $E$ is a complex-valued quantity describing the envelope of the optical field and encompasses 
all amplitude and phase modulation effects imposed by the injected-laser system; $E$ is normalised such that $|E|^{2}$ represents the photon density of the laser field. $F_{N}$ denotes stochastic carrier recombination (which will be defined later). The first term on the righthand side of (2) describes the complex gain of the laser field. The gain coefficient is given by $a\left(N-N_{0}\right)$, where $a$ is the differential gain and $N_{0}$ is the carrier density at transparency. The gain coefficient is modified by the nonlinear gain $\varepsilon_{N L} . \tau_{P}$ is the cavity lifetime. Note that lumping the photon lifetime with the gain-phase coupling allows for the slave laser to be centred at the zero frequency within the simulation; this is beneficial so that master-slave detuning can be easily controlled via the $\exp \left(j 2 \pi f_{D} t\right)$ frequency-translation operation term for the external injection. $E_{i n j}$ is the envelope of the optical field of the master laser; in this paper, we take a constant amplitude (noiseless) for each simulation run. The final term, $F_{E}$, is the random addition of SE due to bimolecular recombination into the lasing field, denoting spontaneous emission into the lasing field.

Table 1. Parameters and values used in the simulation.

\begin{tabular}{ccc}
\hline Symbol & Definition & Value and/or Unit \\
\hline$N$ & Carrier density & $\mathrm{m}^{-3}$ \\
$E$ & Optical field & $\mathrm{m}^{\frac{3}{2}}$ \\
$I$ & Laser bias current & $60 \mathrm{~mA}$ \\
$e$ & Quantum of electronic charge & $1.6 \times 10^{-19} \mathrm{C}$ \\
$V$ & Volume of active region & $6 \times 10^{-17} \mathrm{~m}^{3}$ \\
$A$ & Non-radiative carrier recombination rate coefficient & $1 \times 10^{9} \mathrm{~s}^{-1}$ \\
$B$ & Bimolecular recombination rate coefficient & $1 \times 10^{-16} \mathrm{~m}^{3} \mathrm{~s}^{-1}$ \\
$C$ & Auger recombination rate coefficient & $1 \times 10^{-41} \mathrm{~m}^{6} \mathrm{~s}^{-1}$ \\
$a$ & Differential gain & $9 \times 10^{-13} \mathrm{~s}^{-1} \mathrm{~m}^{3}$ \\
$\Gamma$ & Confinement factor & 0.3 \\
$\alpha_{H}$ & Linewidth enhancement factor & 4 \\
$N_{0}$ & Carrier density at transparency & $1 \times 10^{24} \mathrm{~m}^{-3}$ \\
$\tau_{P}$ & Photon lifetime & $3 \mathrm{ps}^{-1}$ \\
$k_{c}$ & Coupling of external injection into the slave laser & $2 \times 10^{12} \mathrm{~s}^{-1}$ \\
$\beta$ & Fraction of spontaneous emission into the lasing mode & $1 \times 10^{-4}$ \\
$\varepsilon_{N L}$ & Nonlinear gain coefficient & $1 \times 10^{-23} \mathrm{~m}^{3}$ \\
$\Delta f_{D}$ & Master -slave detuning & $\mathrm{Hz}$ \\
$\Delta t$ & Simulation timestep & $1 \mathrm{ps}$ \\
$B_{s i m}$ & Simulation bandwidth & $1 \mathrm{THz}$ \\
$v_{0}$ & Lasing frequency & $193 \mathrm{THz}$ \\
$A$ & Area of lasing mode & $1 \times 10^{-13} \mathrm{~m}^{2}$ \\
$n_{g}$ & Group index & 3.5 \\
\hline
\end{tabular}

${ }^{1}$ Inverse of the simulation timestep.

The stochastic terms are appropriately scaled for numerical computations (1) and (2) with $B_{s i m}=t_{s}^{-1}$, where $t_{s}$ is the step time. We solve the system of equations using Huen's predictor-corrector method.

$$
\begin{gathered}
F_{N}=\sqrt{2 R(N) B_{\text {sim }}} e_{N}(t) \\
F_{E}=\sqrt{\beta B N^{2} B_{\text {sim }}}\left(e_{E I}(t)+j e_{E Q}(t)\right)
\end{gathered}
$$

Each $e$ term is an independent identically distributed random sample taken from a Gaussian random number generator with unity variance. The current is held constant; the only sweeping parameters that we consider is the power of the master laser and the master-slave detuning. We define the detuning $f_{D}=v_{\text {master }}-v_{\text {slave }}$. When solving the equations, we are adjusting $f_{D}$ directly in (2) and the spectrum is always centred for the slave; however, centring the spectrum for the master laser makes it easier to unequivocally show the injection-locking. To centre the spectrum for the master, one needs to take the complex-conjugate of the $\exp \left(2 \pi f_{D} t\right)$ array (calculated when constructing the injection 
term in (4)) and multiply by the output from solving the differential equations $E(t)$ to frequency translate $E(t)$ to be centred at the master laser.

Converting from photon density to optical power $P_{W}$ is given by

$$
\frac{P_{W}}{|E|^{2}}=\frac{c}{n_{g}} h v_{0} A
$$

where $c$ is the speed of light in a vacuum, $n_{g}$ is the group index, $h$ is Planck's constant, and $A$ is the area of the laser mode. One can interpret the spectral scan as essentially tuning the slave laser into the master laser and noting the spectrum. Practically, one would tune the slave laser using temperature control, taking advantage of the available $\sim 0.1 \mathrm{~nm} / \mathrm{K}(12 \mathrm{GHz} / \mathrm{K}$ at $1550 \mathrm{~nm})$ thermal tuning of semiconductor lasers. In all of our simulations, we simulate at a $1 \mathrm{ps}$ timestep, using an initial condition of $N_{I C}=1 \times 10^{24}$ and $E_{I C}=1 \times 10^{12}$. In order to lessen the strength and duration of the transient, we are simulating $E$ by taking 110,000 sample points (unless otherwise stated), and the first 10,000 samples are discarded to remove any transient. The remaining 100,000 data points are used to calculate the spectrum using fast Fourier transforms (FFT). The number of data points and the sampling time allows us a resolution bandwidth of $10 \mathrm{MHz}$, and this is within the range of high-resolution optical spectral analysers. The spectrum we are calculating is the squared magnitude of the (complex valued) FFT array, and here we are circularly shifting by half of the number of samples in the FFT array such that the spectrum is centred at the zero frequency. In order to minimise randomness within the spectra, we perform the following: for each set of parameters the simulation is run twenty times and the spectrum is averaged over those twenty runs. This averages the noise to the average spectral power within each frequency bin in the FFT array.

Preliminary simulations showing the standard laser power of the slave, without external injection, versus bias current are shown in Figure 2a; the threshold current is about $15 \mathrm{~mA}$. The optical spectrum of the slave is shown in Figure $2 \mathrm{~b}$ with a linewidth of $2 \mathrm{MHz}$. The relative intensity noise is show in Figure 2c; the noise at low frequencies is $-165 \mathrm{~dB} / \mathrm{Hz}$ and the relaxation oscillation frequency (ROF) occurs at $9.4 \mathrm{GHz}$.
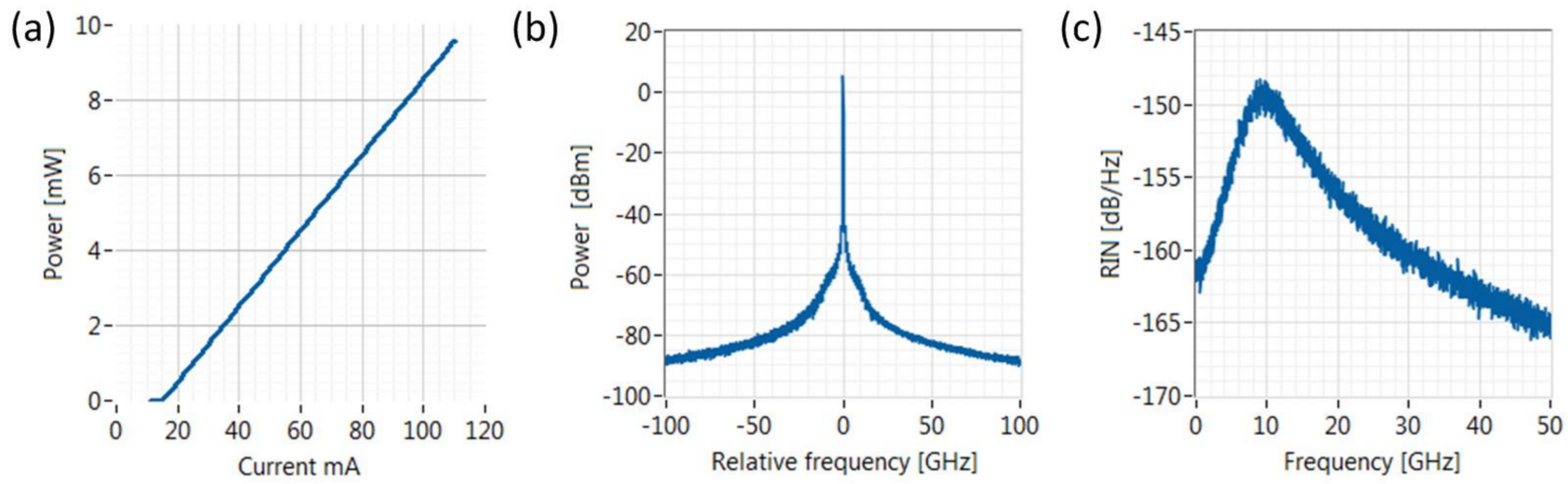

Figure 2. Plot of (a) laser L-I curve of the slave without external injection. (b) The optical spectrum of the free-running slave laser showing Lorentzian broadening. (c) RIN spectrum of the slave laser without external injection showing a relaxation oscillation frequency of $9.4 \mathrm{GHz}$.

\section{High-Resolution Spectral Scans Due to External-Injection}

We show the detailed scans of the output spectra as the slave laser is tuned across the master laser, as was performed experimentally in [18] (Figure 4). To build up the scans, we simulate the averaged spectrum for a given set of laser parameters; then, we adjust one of the parameters (here, we adjust the detuning) and then stack all the calculated spectra and display the spectra as a 3D colour map. Even though our calculated spectra run from $-500 \mathrm{GHz}$ to $500 \mathrm{GHz}$, we only show the spectra running from $-100 \mathrm{GHz}$ to $100 \mathrm{GHz}$ for clarity because the most interesting features of the spectra are located within $\pm 100 \mathrm{GHz}$ of 
the master laser. We plot the spectral scan with the injected power of the master taken to be constant within each scan. The spectral maps are shown in Figures 3 and 4. Different regimes are identified in Figures 3 and 4 by letters $\mathrm{A}-\mathrm{H}$; individual spectra corresponding to the of the different regimes are shown in Figure 5. Trajectories of the photon density and normalised carrier density (N-P trajectories) for the same points $\mathrm{A}-\mathrm{H}$ are shown in Figure 6. Figure 7 presents the calculated RF spectrum after photodetection of the laser output for the different regimes A-H. We will explain the dynamics in more detail later; first, we qualitatively describe the regions labelled A-H in Figures 3 and 4. In Figure 3a, the injection power is $10 \mu \mathrm{W}$, and we can clearly see that there is a short locking range from about $-3 \mathrm{GHz}$ to $3 \mathrm{GHz}$, when the output of the slave laser is locked to the master laser; when locking has not been achieved, obvious four wave mixing (FWM) products appear in the spectrum. Note that all of the spectra shown in this section are qualitatively similar to the experimentally taken spectra of external injection dynamics in [11].

Case A: FWM. Region A corresponds to FWM between the master and slave, and the master and slave are clearly not locked; the beating between both lasers modulates the slave laser to create additional frequency products in the spectrum, each spaced by $f_{\mathrm{d}}$ about the slave laser. This is clearly shown in the optical spectrum in Figure 3a. The N-P trajectory in Figure 6A for FWM shows a slight modulation of the carrier density (as expected), and the RF spectrum in Figure 7A shows a few peaks each spaced by $f_{\mathrm{d}}$.

Case B: Injection-locking. When the detuning between the two lasers is reduced, the magnitude of the varying carrier density increases and becomes sufficient to synchronise the slave laser to the master laser. The lasing frequency of the slave also reduces because the carrier density for threshold of the injected laser system is smaller than that for the solitary free-running laser system. Injection-locking has been extensively studied previously [1-13] and we shall only briefly describe the results. The N-P trajectories (Figure 6B) indicate a single point for the injection-locked case and negligible modulation of the photon and carrier densities. We can deduce the carrier density at threshold of $N_{t h} 1.42 \times 10^{24} \mathrm{~m}^{-3}$ (injection power of $10 \mu \mathrm{W}$ ), which is lower than the carrier density at threshold for the solitary slave laser of $1.422 \times 10^{24} \mathrm{~m}^{-3}$. When $N$ exceeds (goes below) the threshold, the slave becomes amplifying (attenuating). The optical spectrum shows a single lasing mode, and the absence of strong mixing tones in the RF spectrum (Figure 7) indicates that the system is locked.

Increasing the injection power exacerbates the modulation of the photon and carrier densities; therefore, we expect more complex and interesting behaviour. As the injection power is increased, the locking range is increased, as is the case in Figure 3b. We notice, here, that for detuning values close to the locking range, the slave is pulled more strongly towards the master. The next dynamical feature that appears is FWM-induced period-doubling.

Case C: FWM-induced period-doubling. This feature appears when the FWM goes into a period-doubling type oscillation for detuning close to the locking range before injectionlocking occurs. In this example, there is detuning of about $-18 \mathrm{GHz}$ (Figure $4 \mathrm{a}$ and it is also observed in [18] (Figure 4). The cause of this behaviour is that the RO frequency of the slave is midway between the master and slave detuning; hence, the pulsation arising from the FWM exacerbates period-doubling oscillation. As the detuning is increased, the slave laser emerges into a FWM regime without the laser going into self-pulsation.

Case D: Self-pulsation. As the master power is increased further, regions of selfpulsation appear, as shown at point $\mathrm{D}$ in Figure $4 \mathrm{a}$. This happens because the system becomes unstable as the damping can no longer suppress random fluctuations in the photon density; hence, optical pulsations grow. One can clearly see that the spectral lines of the self-pulsation coincide with the ROF. The laser system cannot support continuously growing oscillation and the oscillating pulses deplete the carrier density and the pulses thus decay, thereby keeping the self-pulsation stable. The process keeps repeating because the carrier density builds up in the absence of photons, and once the pulse builds up, this depletes the carrier density. In Figure 6D, by looking at the N-P trajectory, one can see a large swing in the carrier density in addition to a large swing in the photon density. 


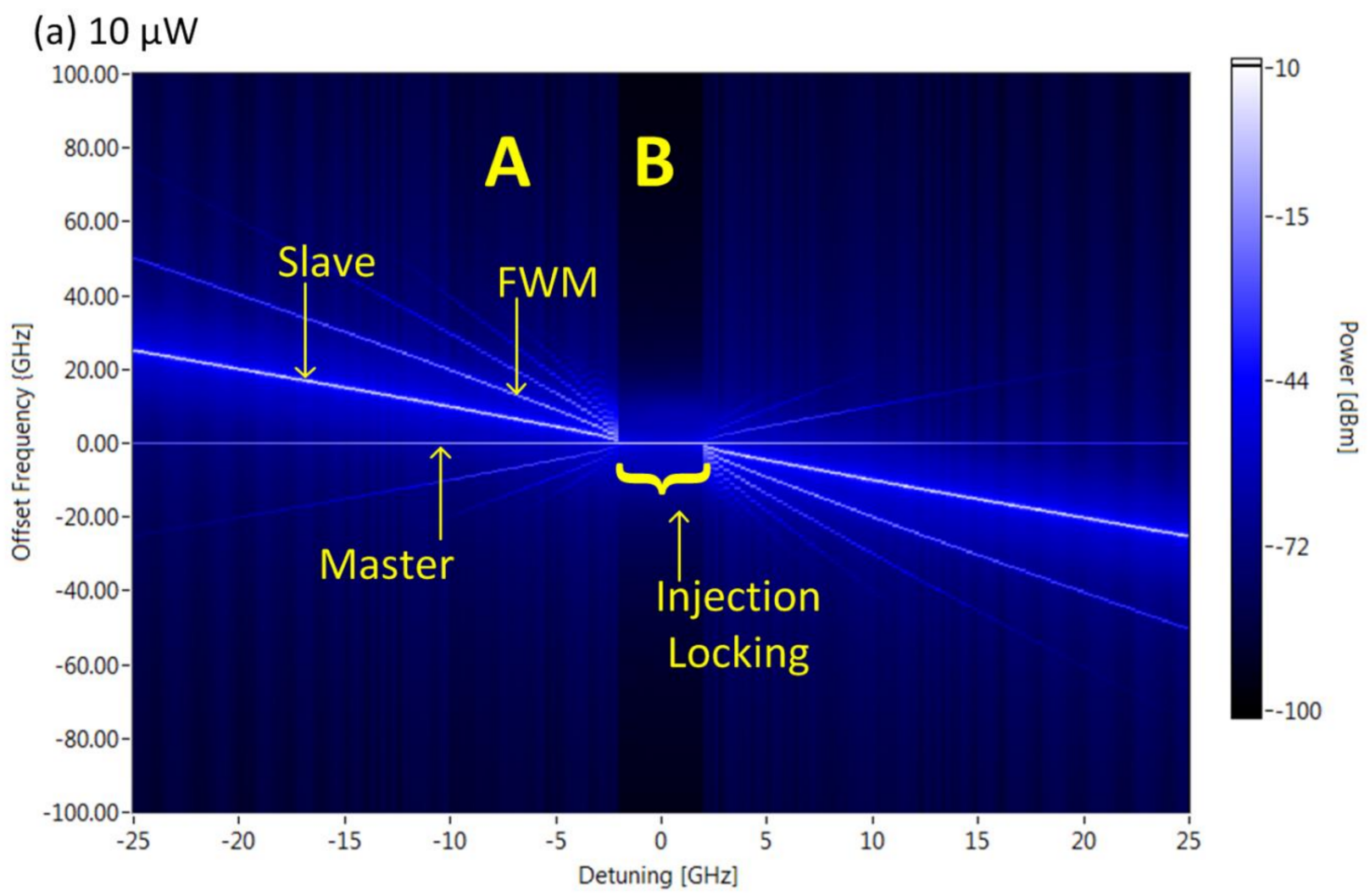

(b) $100 \mu \mathrm{W}$

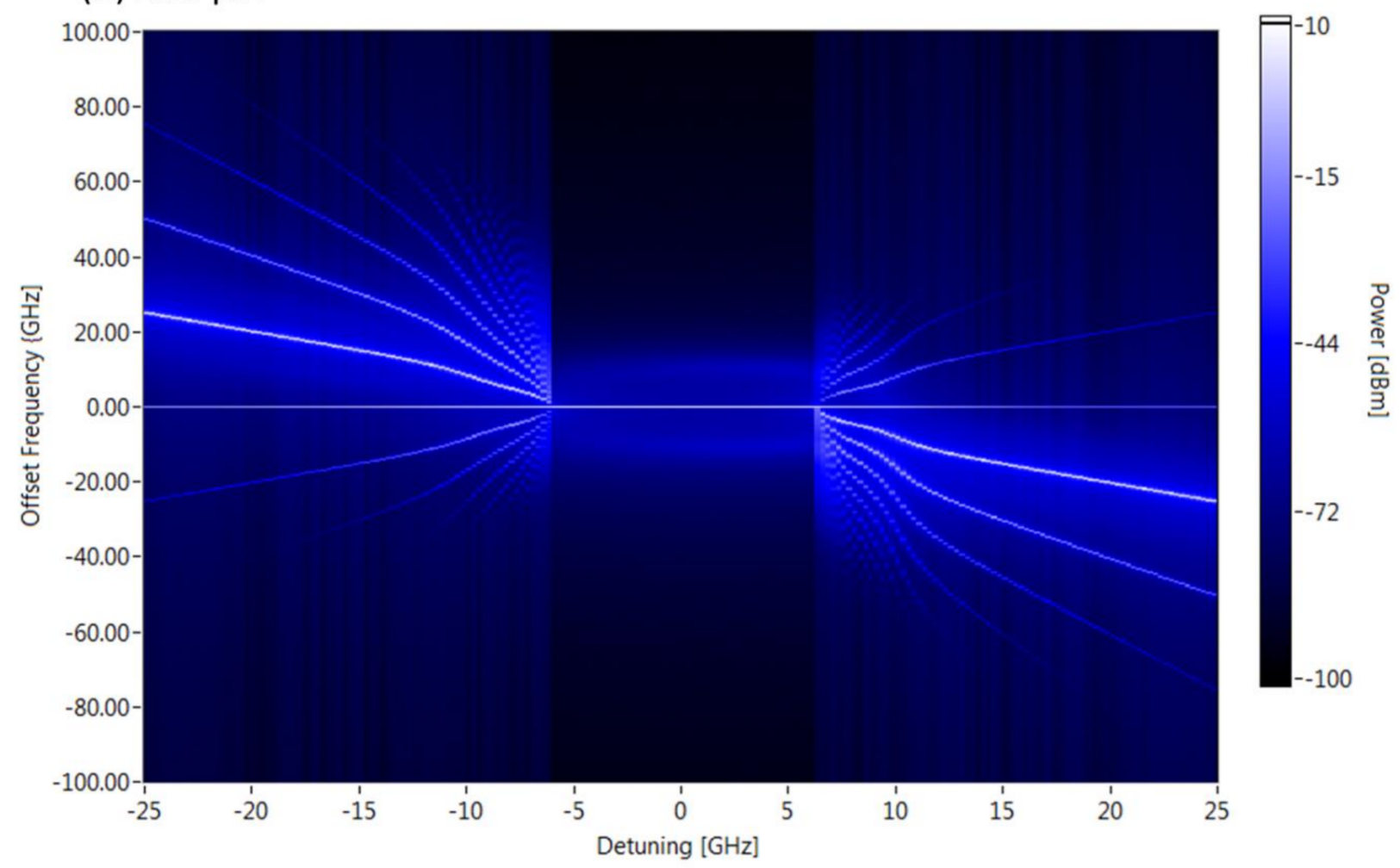

Figure 3. Simulation of the spectra as the master slave detuning is increased from $-25 \mathrm{GHz}$ to $25 \mathrm{GHz}$. Each subplot shows the scan for a different master power. The master power is set to (a) $10 \mu \mathrm{W}$, (b) $100 \mu \mathrm{W}$. The different regions are identified in yellow capitals: A is FWM; B is injection-locking. 


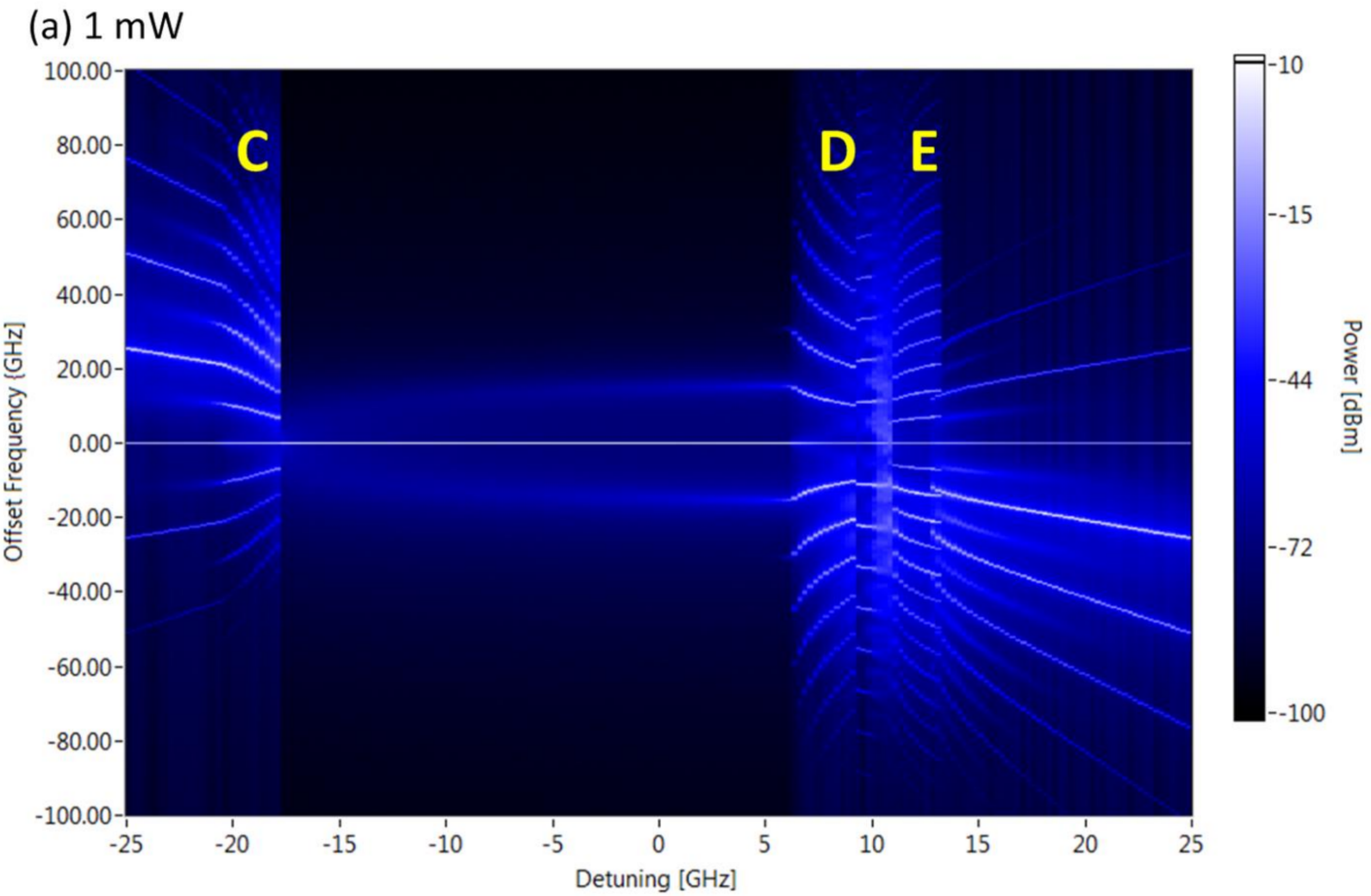

(b) $2 \mathrm{~mW}$

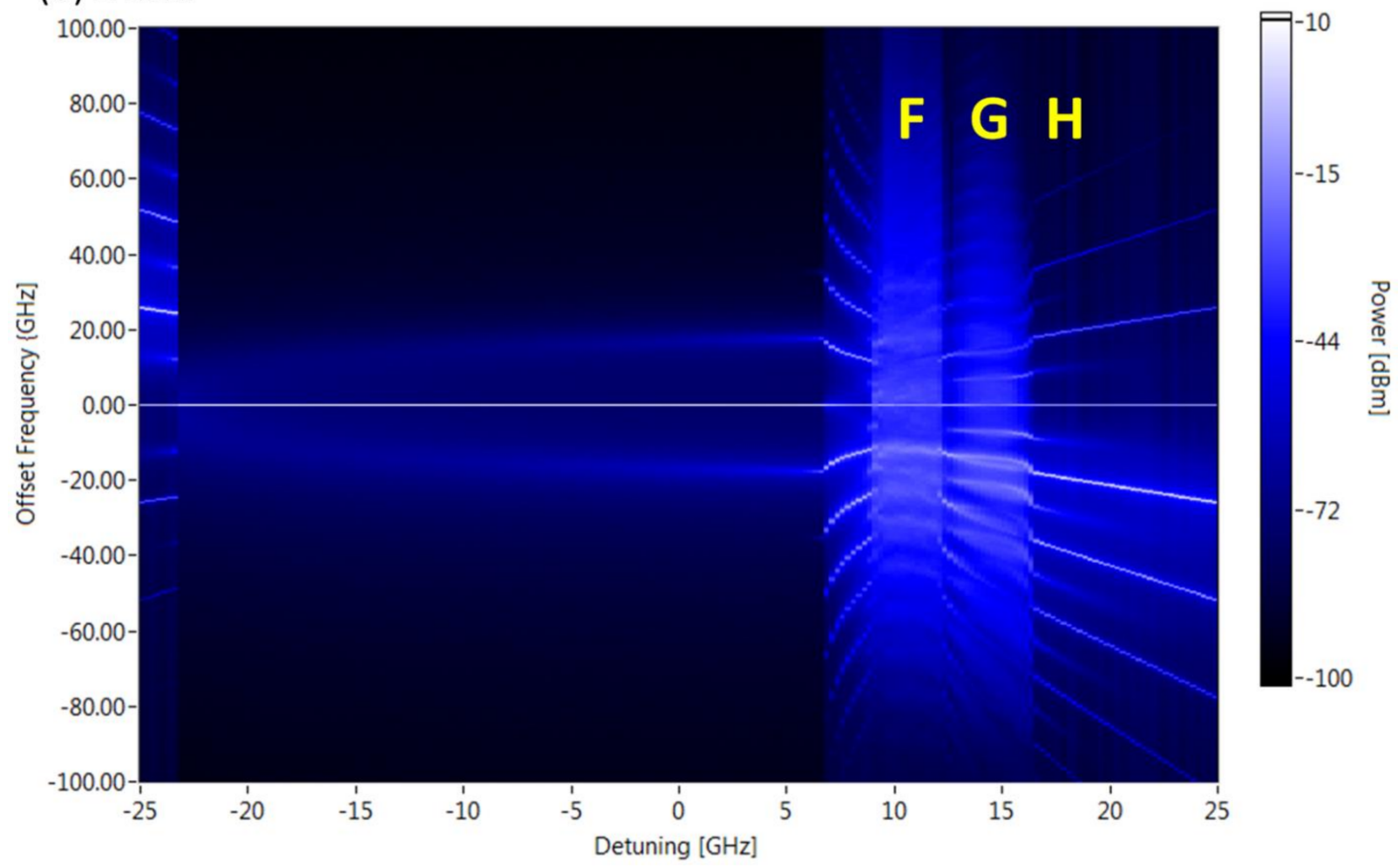

Figure 4. Simulated detuning scan of the spectral evolution of the externally injected laser as the injection power is increased to (a) $1 \mathrm{~mW}$ and (b) $2 \mathrm{~mW}$. In these plots, the labelled regions are: (C) is a frequency doubled pulsation due to FWM as the laser nears injection-locking regime, self-pulsation (D), period-doubling (E), chaos (F), (G) is chaos with period-doubled oscillations and subject to a more detailed study in Section 4; $(\mathrm{H})$ is another FWM regime. 

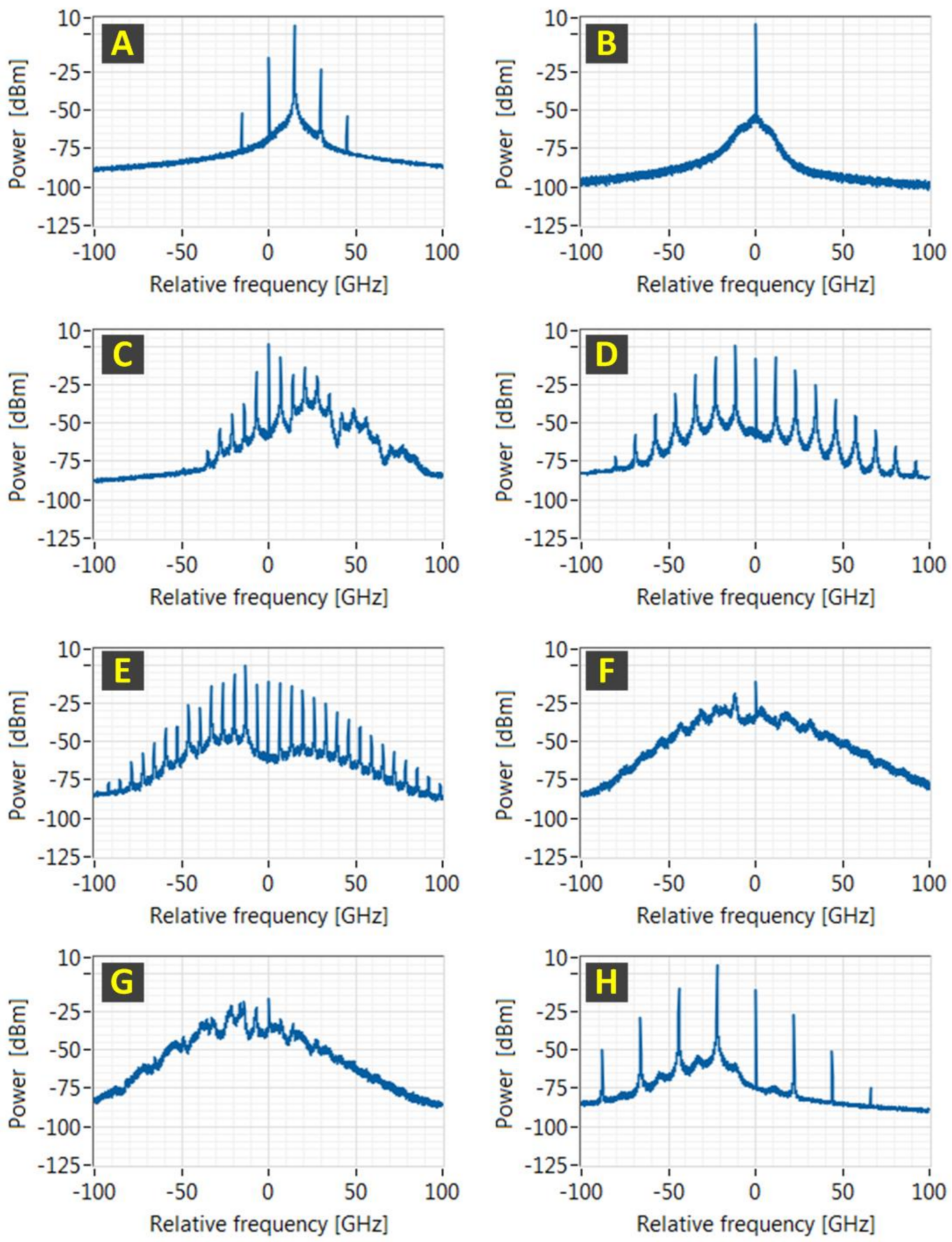

Figure 5. Averaged optical spectra corresponding to the regimes $(\mathbf{A}-\mathbf{H})$ in Figures 3 and 4 . One could consider Figures 3 and 4 to be a stacked collection of these spectra. The pulsation regimes (D) are the P-1 oscillation regimes, whereas (E) is the P-2 oscillation regime. The chaotic regimes in $(\mathrm{F}, \mathrm{G})$ show wide spectrum though a clear absence of any spectral line structure that would indicate regular pulsation. 

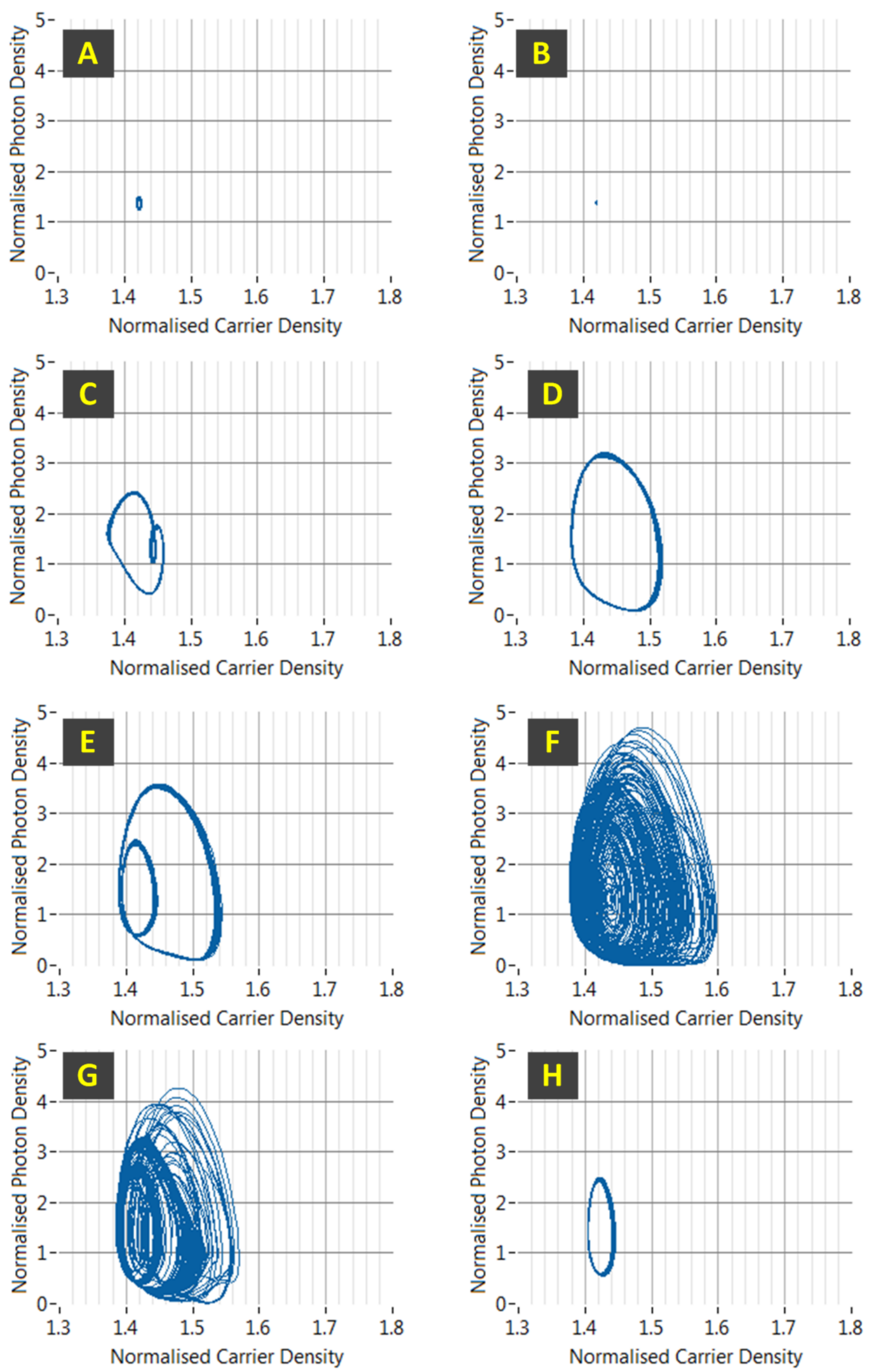

Figure 6. Phase-space portraits of the trajectory of the carrier and photon densities for the regimes outlined in Figure 1. Slight modulation for the FWM states in $(\mathbf{A}, \mathbf{H})$, a static point when injectionlocked in (B). Period-doubling in (C,E); note the position of the extra loop in both cases for P-1 pulsations (D) and chaos in $(\mathbf{F}, \mathbf{G})$. 

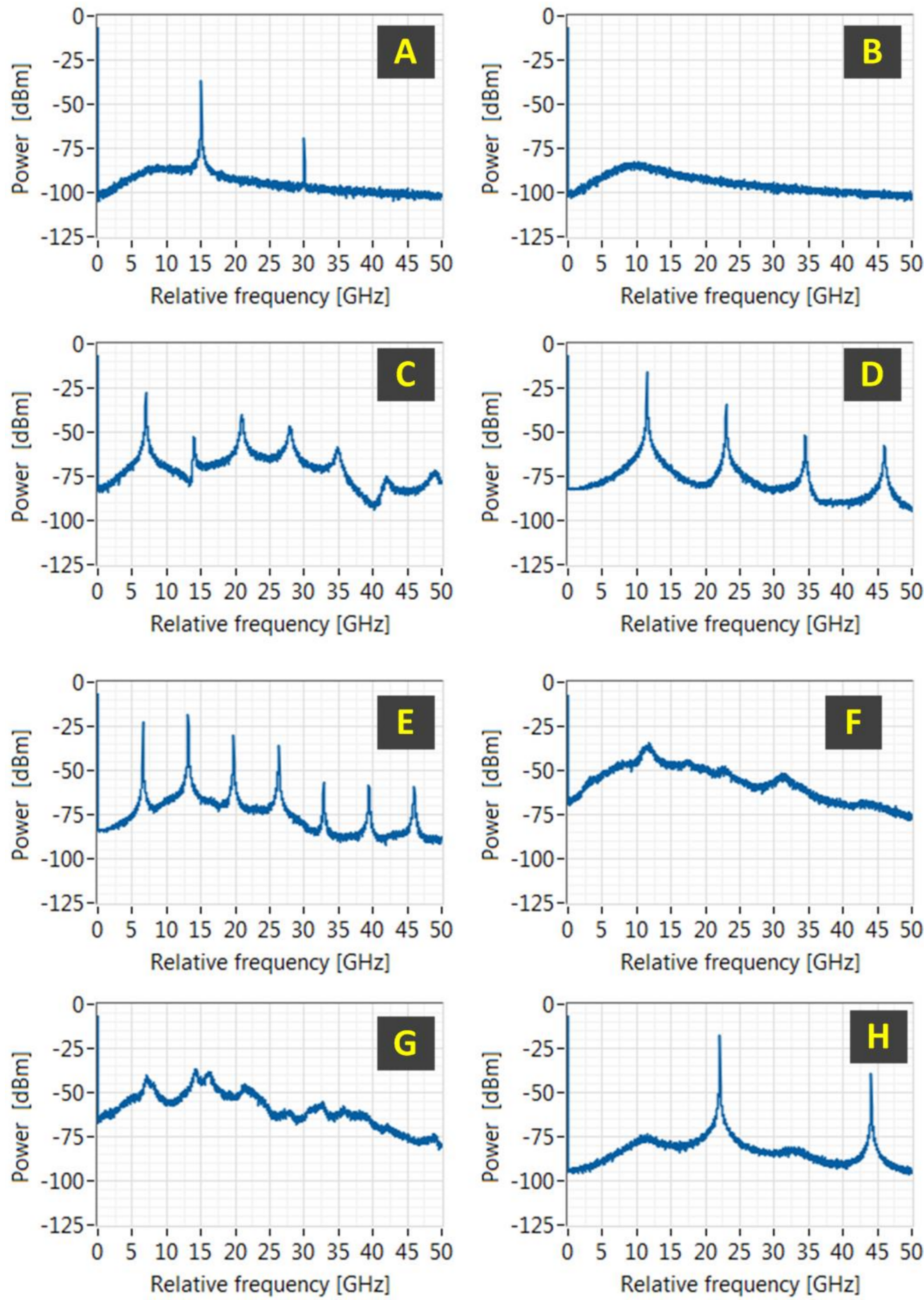

Figure 7. Calculated RF spectra of the regimes $(\mathbf{A}-\mathbf{H})$ identified in Figures 3 and 4. Injection-locking is achieved when the RF spectrum is minimised. The RF spectrum can yield information about the pulsation regimes, allowing one to distinguish between pulsation regimes (C-E) showing clear spectral lines and chaotic pulsation regimes $(\mathbf{F}, \mathbf{G})$ showing a broad continuum. The RF spectra for the FWM cases $(\mathbf{A}, \mathbf{H})$ show a few tones in the spectrum with the tone spacing equalling the master-slave detuning frequency.

Case E: Period-doubling. When the photon and carrier densities require two oscillation periods to revert to the same position, extra spectral lines, which are spaced at half the distance for the case of self-pulsation spectral lines, also appear in the spectrum in region $\mathrm{E}$ in Figure $4 \mathrm{a}$ and within the optical and RF spectra in Figures 5E and 7E, respectively. In the trajectory in Figure 6E, a small loop appears, indicating a pattern of consecutive 
larger and smaller pulses in the pulse train. One interesting aspect to note that is indicated by the simulations is that there is an obvious narrowing of the spectral lines for perioddoubling (Figure 5E compared to the spectral lines self-pulsation case (Figure 5D). This can be explained by looking at their N-P traces in Figure 6E,D, respectively. One can clearly see that the photon density tends towards zero in both scenarios; large phase changes occur when the value of the laser field is small compared to the SE [19]. For the self-pulsation case, the photon density goes to zero once per pulsation cycle; however, for the period-doubling case, the photon density goes to zero once at half the rate for self-pulsation. This explains why the spectral line broadening is smaller for period-doubling compared to self-pulsation

Case F: Chaos. Chaos has been studied in detail before [6,7], so we will just briefly describe the effect here. The main characteristic of chaos is the broad continuous spectrum; this is clearly seen in Figures $4 \mathrm{~b}$ and $5 \mathrm{~F}$. As the injection power is increased to $2 \mathrm{~mW}$, then it is possible for the pulsations to become completely chaotic, as there is no way to regularise the photon density in the cavity at the instant the carrier density reaches above the threshold. Looking at the detuning range at which chaotic behaviour is observed in Figure $4 \mathrm{~b}(\sim 10 \mathrm{GHz}$ detuning), and noting the corresponding region in Figure $4 \mathrm{a}$ when less injection power is used: in that detuning region $(\sim 10 \mathrm{GHz})$ in Figure $4 \mathrm{a}$, notice there is an abrupt change in the spectral line spacing when increasing the detuning in region $\mathrm{D}$ in Figure 4a; it is within this region of abrupt pulsation frequency change that chaos occurs when the injection power is increased. It is interesting to note from the N-P trajectory of chaotic regime in Figure $6 \mathrm{~F}$ that there is no regular pulsation, as is the case for the trajectory for self-pulsation; however, in the coherence case, photon density can go towards zero, and the time taken to build up a lasing field again depends on the value of the photon density in the slave laser as the laser goes above the threshold. One curious aspect of chaos is that the optical spectrum is the same irrespective of whether or not SE is included in the simulation. We have described in previous work how regular pulse trains can have continuous optical spectra without any 'comb'-like structure when there is no memory of the optical phase from pulse to pulse [20]. We rule out any such phenomenon being the cause of the continuous spectrum for chaos by looking at the RF spectrum in Figure 7F; there are clearly no distinct lines that would indicate pulsation with jitter [21].

Case G: Noise-Induced Chaos Regime. This is the regime that we are identifying for the first time in this paper. It is clear in Figure $5 \mathrm{G}$ that the laser system is going into a different pulsation regime to that of chaos in Case $\mathrm{F}$ (Figure 5F). The extra peaks in the spectrum, similar to those of period-doubling, are visible, though the laser is still in a chaotic state. As we show in the following section, without SE, this region is comprised of a higher-order pulsation regime. Clearly, the N-P trajectory is chaotic (Figure 6G); moreover, the chaotic trajectory looks to have the same form for period-doubling in Figure $6 \mathrm{E}$, though there is no regular trajectory path and it is clearly taking a chaotic trajectory.

Case H: Four-wave mixing. As the master-slave detuning is increased further, the lasers no longer pulsate because of the limited carrier density dynamics, and only FWMproducts are created, which is similar to case A.

Now that we have described all of the operating regimes of the externally injected laser system, we concentrate on case $G$, the period-doubling regime, in more detail.

\section{Influence of Spontaneous Emission}

In this section, we justify the claim made in the previous section that the SE is responsible for keeping the injected laser system, for high injection powers, in a chaotic state. To enact this, we repeat the scans of Figure $4 a, b$ with the value of the spontaneous coefficient $\beta$ set to zero. The results are plotted in Figure 8 for injection powers of (a) $1 \mathrm{~mW}$ and (b) $2 \mathrm{~mW}$. For the case of $1 \mathrm{~mW}$ injection without SE, a very thin region of chaos is clearly identifiable by a spectral continuum at a detuning of about $10.5 \mathrm{GHz}$. Note this was present in Figure 4a, though the detuning range over which chaos occurs here is narrow; thus, we concentrated on chaos at the higher power of $2 \mathrm{~mW}$ for clarity. In Figure 8a, clear evidence of P-2, P-3 and P-4 oscillations appears either side of this narrow chaotic state 
(in the vicinity of detuning of $11 \mathrm{GHz}$ ), which were not present when SE was included in Figure 4a; this finding is consistent with [14], where a lack of a period-doubling route to chaos was found. Within this detuning window in Figure 8a, periodic-pulsation states are now observable due to the removal of SE in the model. This noise-induced chaos behaviour was formally studied in [15]. The impact of SE is starker for the second chaotic region in Figure $4 \mathrm{~b}$. There are now clearly observable P-2, P-3 and P-4 oscillations within the detuning window from $13 \mathrm{GHz}$ to $17 \mathrm{GHz}$; these are washed away by the SE in Figure $4 \mathrm{a}$, where a chaotic state with the hallmarks of a P-2 state exists over the detuning range from 13 to $17 \mathrm{GHz}$ (approx.).

(a) $1 \mathrm{~mW}$ injection, without SE

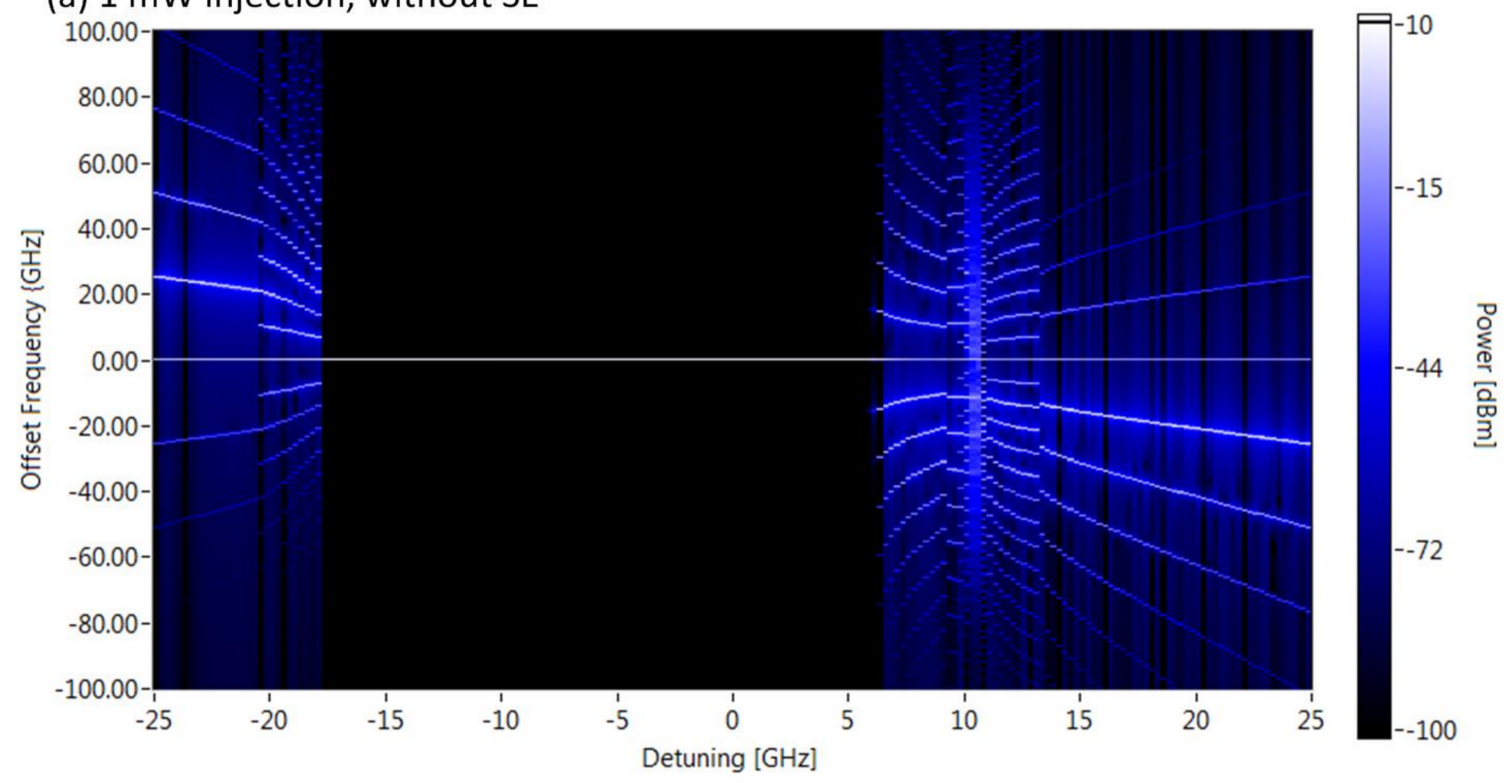

(b) $2 \mathrm{~mW}$ injection, without SE

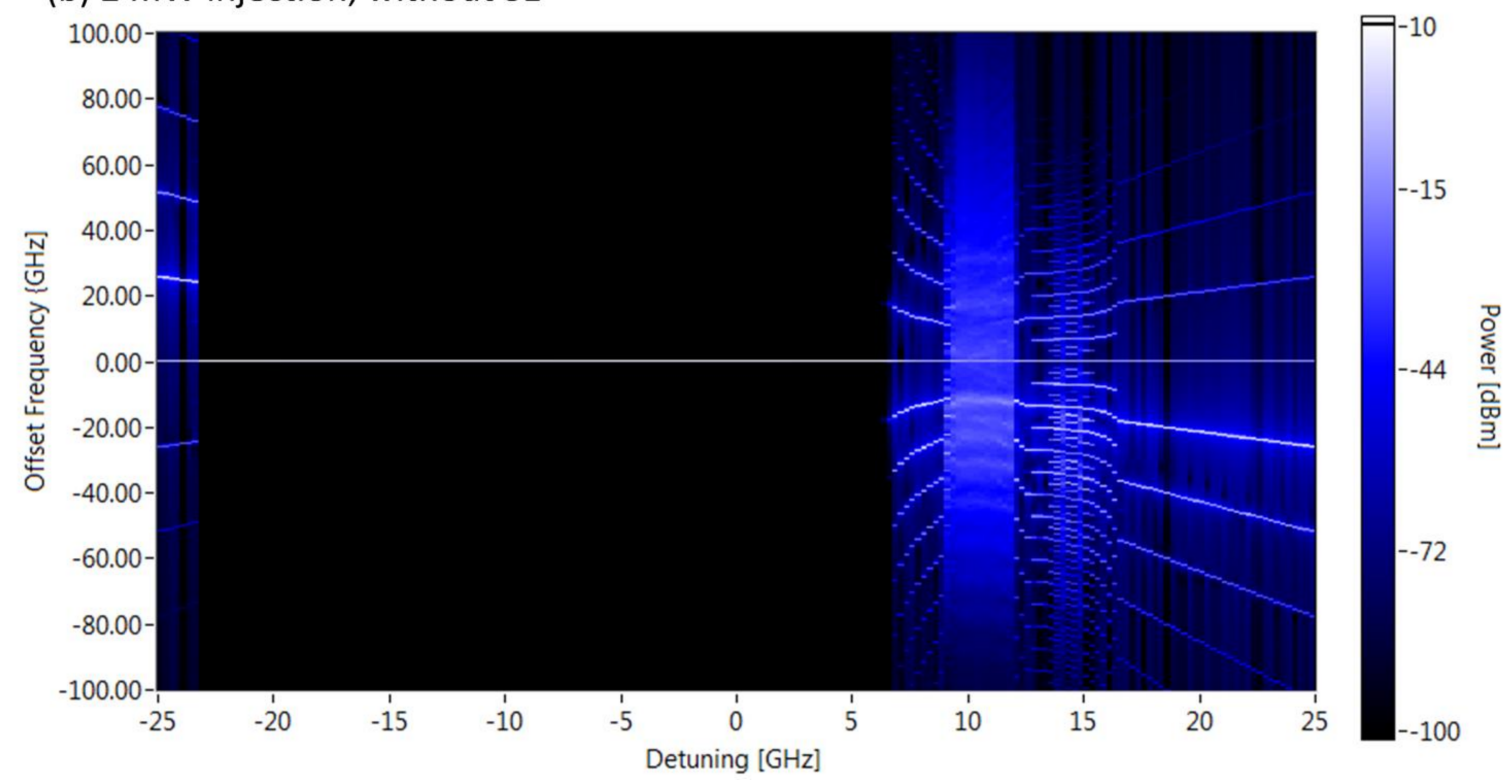

Figure 8. Scan of the injection-locking for (a) $100 \mathrm{uW}$ and (b) $200 \mathrm{uW}$. In each case, the value of the spontaneous emission coefficient is set to zero. These plots show more dynamical pulsation states than in Figure 4, especially for the case of $2 \mathrm{~mW}$ in the region from 6 to $20 \mathrm{GHz}$ detuning.

The detail in these scans is only as good as the detuning granularity when constructing the spectral scans with detuning. A more in-depth examination at the spectral plots is 
repeated by exploring the detuning over smaller detuning ranges, as well as zooming into the central portion of the spectrum. Spectral resolution is also enhanced by increasing the number of samples taken when solving the system equations; the number of samples taken was 1,010,000 (the first 10,000 samples are discarded). The spectral resolution is now $1 \mathrm{MHz}$. These were conducted over the detuning range from 6 to $18 \mathrm{GHz}$ with the injection power equal to $2 \mathrm{~mW}$; the spectral scan results are shown in Figure 9. This figure clarifies the stark differences when SE is included or omitted in the simulations, and higher-order P5 (and above) oscillations are revealed when SE is omitted.

\section{(a) Without SE}

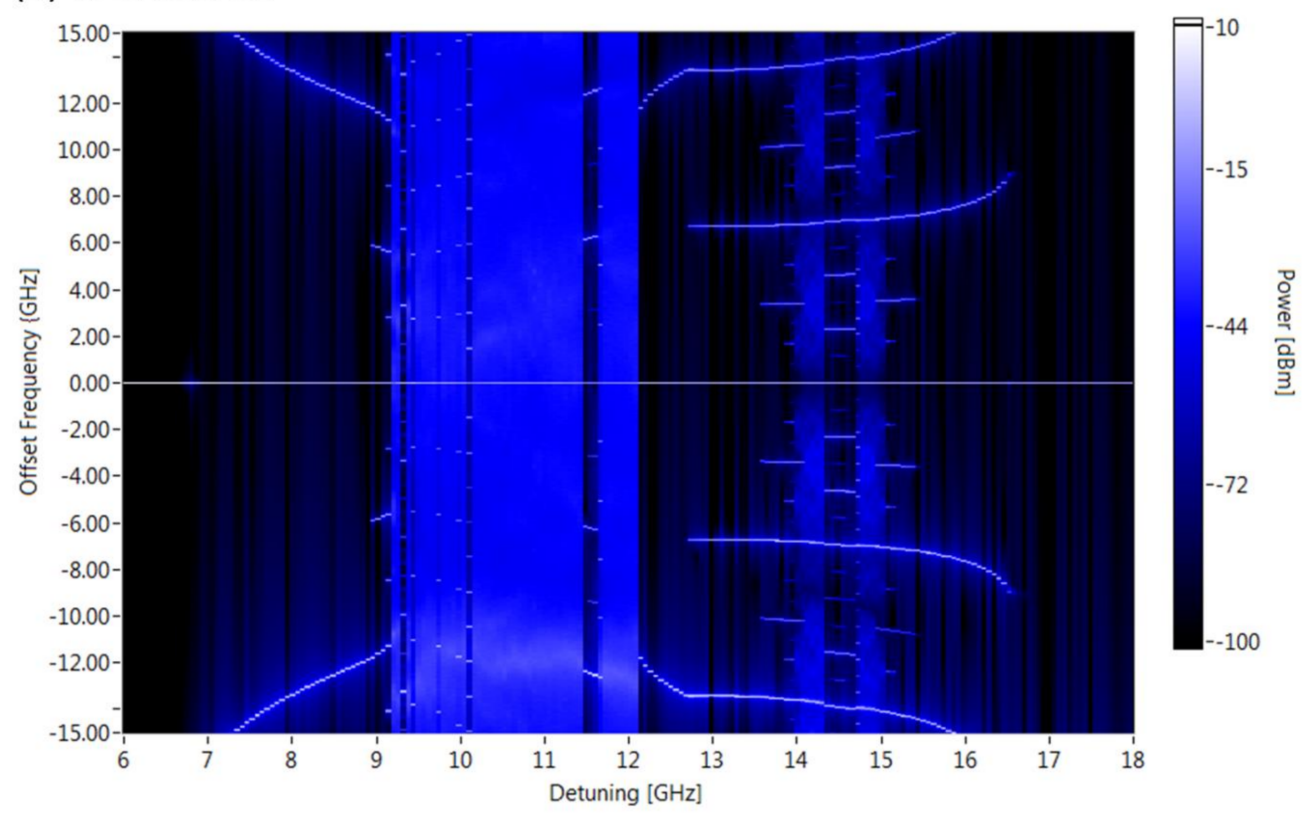

(b) With SE Included

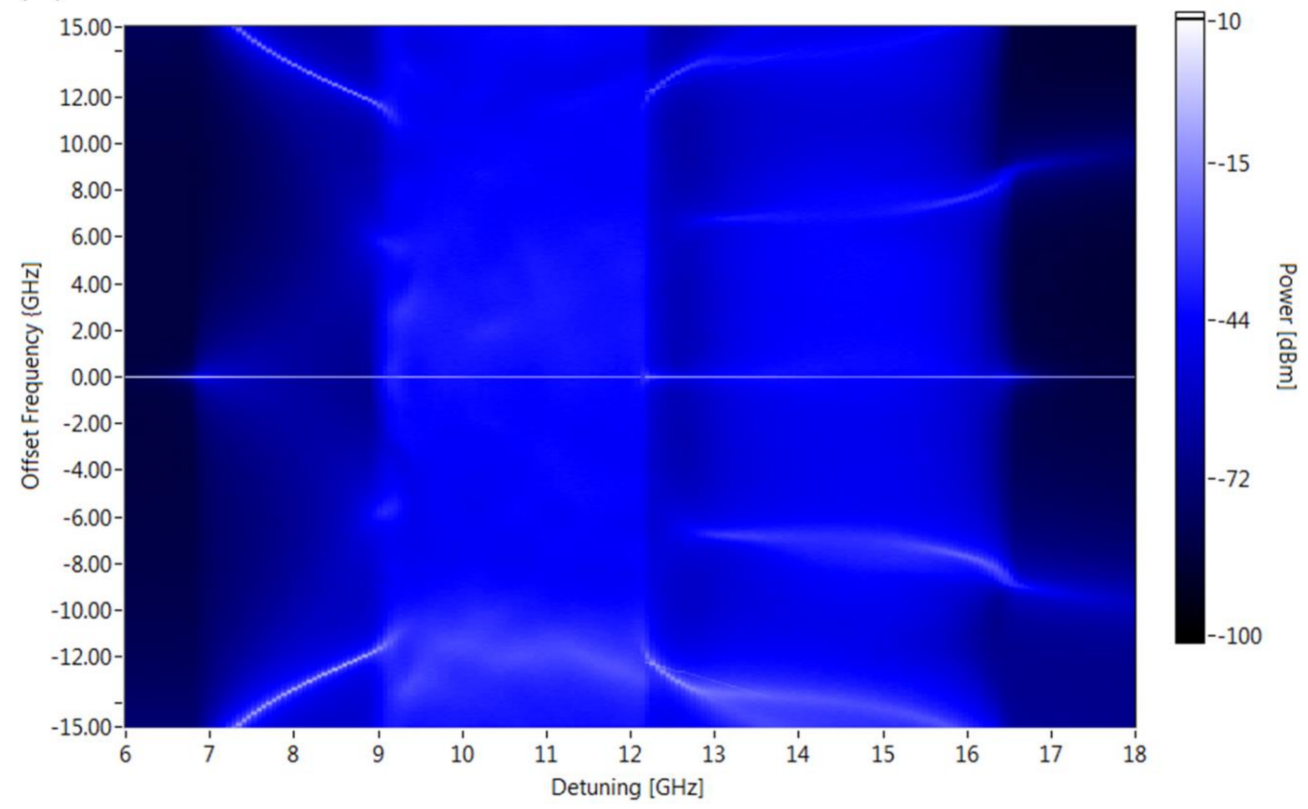

Figure 9. Detailed scan of the region around the chaotic regime showing the changes from regular pulsations to chaotic pulsations for the case (a) without SE and (b) including SE. The injection power is $2 \mathrm{~mW}$. Note the absence of period-N oscillations when SE is included, leaving just the amalgamated chaotic version of the P-2 state for detunings around $14 \mathrm{GHz}$ to $15 \mathrm{GHz}$. 
For completeness, we investigate the level of SE coupling required to observe oscillations of a higher order than P-2 oscillations. We take the injection power level to be $2 \mathrm{~mW}$ and the master-slave detuning to be $14.55 \mathrm{GHz}$. In Figure 9, we find that there is a P-6 oscillation without SE and a noise-induced chaotic state with SE included. We find that the SE coupling needs to be reduced below the value of $1 \times 10^{-7}$ to see the P-6 oscillation; the scan of the spectra with the value of SE coupling increased from 0 to $1 \times 10^{-7}$, as shown in Figure 10.

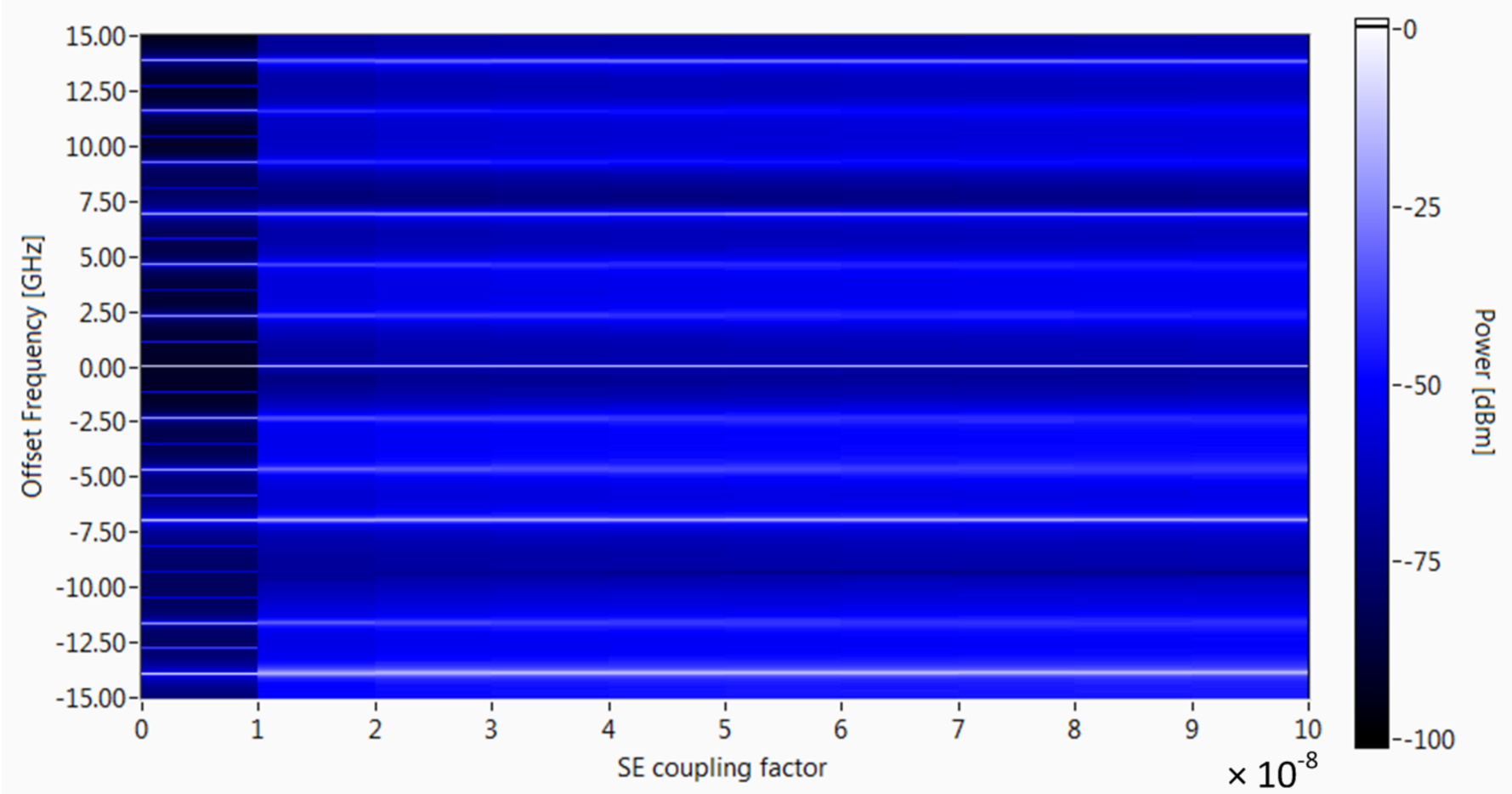

Figure 10. Spectral scan of the influence when very low SE coupling for the external injection laser system when the detuning is $14.55 \mathrm{GHz}$ and the injection power is $2 \mathrm{~mW}$. At the right-hand side of the plot, we note a continuum in the spectrum with two prominent horizontal lines corresponding to the noise-induced chaos within a P2 pulsation. As the SE coupling is reduced towards zero (far left), we see extra horizontal lines emerge, which signify emergence of a higher-order pulsation period. When SE coupling equals zero, there are three extra spectral lines between the P2 pulsation lines; therefore, the system is in a P6 regime. The values of SE coupling are at least 3 orders of magnitude below typical value of SE coupling.

\section{Conclusions}

We have shown, through numerical solutions, the spectral evolution of an externally injected laser system and the role that SE plays in washing out higher-order pulsation regimes in the dynamics of externally injected lasers. We identify an operating regime comprised entirely of noise-induced chaos destroying higher-order pulsations, especially under strong injection powers. The spectral scans by themselves would be helpful to those operating externally injected lasers to understand the regimes of operation as they are tuning the lasers to achieve injection-locking. The level of employed SE in the slave laser would be considered to be at the lower end, as we chose a value of SE coupling coefficient to be an order of magnitude lower than a typical value for this parameter. We show that a value of SE coupling needs to be as low as $1 \times 10^{-7}$, many orders of magnitude smaller than typical values in order to observe higher-order pulsations. There is sufficient experimental evidence to show existence of P3 oscillations in [6,22] and P4 oscillations in $[5,14]$, though much work is needed to understand the laser parameters and operating conditions of 
injection lasers to achieve higher-order pulsations than the P2 pulsations in the presence of SE.

Author Contributions: Conceptualization, S.P.O. and L.P.B.; methodology, S.P.O.; software, S.P.O.; validation, S.P.O. and L.P.B.; formal analysis, S.P.O. and L.P.B. writing-original draft preparation, S.P.O.; writing-review and editing, L.P.B.; funding acquisition, L.P.B. All authors have read and agreed to the published version of the manuscript.

Funding: This work has emanated from research supported in part by a research grants 18/EPSRC/3591 and 12/RC/2276_P2 from Science Foundation Ireland (SFI); co-funded under the European Regional Development Fund.

Acknowledgments: S.O.D. is grateful to Prince Anandarajah of Dublin City University for fruitful discussions. The authors are grateful to two anonymous reviewers for pointing out important prior works on this topic.

Conflicts of Interest: The authors have no conflict of interest to declare.

\section{References}

1. Lang, R. Injection locking properties of a semiconductor laser. IEEE J. Quantum. Electron. 1983, 18, 976-983. [CrossRef]

2. Annovazzi-Lodi, V.; Scire, A.; Sorel, M.; Donati, S. Dynamic behavior and locking of a semiconductor laser subjected to external injection. IEEE J. Quantum Electron. 1998, 32, 2350-2357. [CrossRef]

3. Schunk, N.; Petermann, K. Noise analysis of injection-locked semiconductor injection lasers. IEEE J. Quantum Electron. 1986, 22, 642-650. [CrossRef]

4. Liu, Z.; Slavík, R. Optical injection locking: From principle to applications. IEEE J. Lightw. Technol. 2019, 38, 43-59. [CrossRef]

5. $\quad$ Blin, S.; Guignard, C.; Besnard, P.; Gabet, R.; Stéphan, G.M.; Bondiou, M. Phase and spectral properties of optically injected semiconductor lasers. C. R. Phys. 2003, 4, 687-699. [CrossRef]

6. Wieczorek, S.; Krauskopf, B.; Simpson, T.B.; Lenstra, D. The dynamical complexity of optically injected semiconductor lasers. Phys. Rep. 2005, 416, 1-128. [CrossRef]

7. Lenstra, D.; Verbeek, D.H.; den Boef, A.J. Coherence Collapse in Single-Mode Semiconductor Lasers Due to Optical Feedback Coherence collapse in single-mode semiconductor lasers due to optical feedback. IEEE J. Quantum Electron. 1985, 21, 674-679. [CrossRef]

8. Daly, A.; Roycroft, B.; Corbett, B. Stable locking phase limits of optically injected semiconductor lasers. OSA Opt. Expr. 2013, 21, 30126-30139. [CrossRef]

9. Simpson, T.B.; Liu, J.M.; Gavrielides, A. Bandwidth enhancement and broadband noise reduction in injection-locked semiconductor lasers. IEEE Photon. Technol. Lett. 1995, 7, 709-711. [CrossRef]

10. Lau, E.K.; Zhao, X.; Sung, H.K.; Parekh, D.; Hasnain, C.C.; Wu, M.C. Strong optical injection-locked semiconductor lasers demonstrating 100-GHz resonance frequencies and 80-GHz intrinsic bandwidths. OSA Opt. Exp. 2008, 16, 6609-6618. [CrossRef]

11. Herrera, D.J.; Kovanis, V.; Lester, L.F. Using transitional points in the optical injection locking behavior of a semiconductor laser to extract its dimensionless operating parameters. IEEE J. Sel. Top. Quantum Electron. 2022, 28, 1800109. [CrossRef]

12. Gunning, P.; Lucek, J.K.; Moodie, D.G.; Smith, K.; Davey, R.P.; Chernikov, S.V.; Guy, M.J.; Taylor, J.R.; Siddiqui, A.S. Gainswitched DFB laser diode pulse source using continuous wave light injection for jitter suppression and an electroabsorption modulator for pedestal suppression. Electron. Lett. 1996, 32, 1010-1011. [CrossRef]

13. Anandarajah, P.M.; Maher, R.; Xu, Y.Q.; Latkowski, S.; O'Carroll, J.; Murdoch, S.G.; Phelan, R.; O'Gorman, J.; Barry, L.P. Generation of Coherent Multicarrier Signals by Gain Switching of Discrete Mode Lasers. IEEE Photon. J. 2011, 3, 112-122. [CrossRef]

14. Kovanis, V.; Gavrielides, A.; Simpson, T.B.; Liu, J.M. Instabilities and chaos in optically injected semiconductor lasers. Appl. Phys. Lett. 1995, 67, 2780-2782. [CrossRef]

15. Hwang, S.K.; Gao, J.B.; Liu, M.; Liu, J. Noise-induced chaos in an optically injected semiconductor laser mode. Phys. Rev. E 2000, 61, 5162. [CrossRef]

16. Kajiyama, K.; Hata, S.; Sakata, S. Effects on spontaneous emission on dynamic characteristics of semiconductor lasers. Appl. Phys. 1977, 12, 209-210. [CrossRef]

17. Dúill, S.P.Ó.; Anandarajah, P.M.; Zhou, R.; Barry, L.P. Numerical investigation into the injection-locking phenomena of gain switched lasers for optical frequency comb generation. Appl. Phys. Lett. 2015, 106, 211105. [CrossRef]

18. Zou, L.-X.; Huang, Y.-Z.; Liu, B.-W.; Lv, X.-M.; Long, H.; Yang, Y.-D.; Xiao, J.-L.; Du, Y. Nonlinear dynamics for semiconductor microdisk laser subject to optical injection. IEEE J. Sel. Top. Quantum Electron. 2015, 21, 1800408. [CrossRef]

19. Schawlow, A.L.; Townes, C.H. Infrared and optical masers. Phys. Rev. 1958, 112, 1940. [CrossRef]

20. Dúill, S.P.Ó.; Zhou, R.; Anandarajah, P.M.; Barry, L.P. Analytical approach to assess the impact of pulse-to-pulse phase coherence of optical frequency combs. IEEE J. Quantum Electron. 2015, 51, 1200208. [CrossRef] 
21. Leep, D.A.; Holm, D.A. Spectral measurement of timing jitter in gain-switched semiconductor lasers. Appl. Phys. Lett. 1992, 60, 2451-2453. [CrossRef]

22. Gavrielides, A.; Kovanis, V.; Nizette, M.; Erneux, T.; Simpson, T.B. Period three limit-cycles in injected semiconductor lasers. J. Opt. B Quantum Semiclass. 2002, 4, 20. [CrossRef] 